\title{
Anaesthetic management of an Extremely Low Birth Weight (ELBW) neonate with multiple co-morbidities
}

\author{
Saajan Joshi ${ }^{1}$, Deepak Dwivedi ${ }^{2}{ }^{2,}$, Randeep Kaur ${ }^{3}$, Indu Bala ${ }^{4}$ \\ ${ }^{1,2,3}$ Senior Resident, ${ }^{4}$ Professor, Dept. of Anaesthesia and Intensive Care, Postgraduate Institute of Medical Education and \\ Research, Chandigarh, India
}

*Corresponding Author:

Email: deepakdwivedi739@gmail.com

Received: $10^{\text {th }}$ June, 2017

Accepted: $31^{\text {st }}$ August, 2017

\begin{abstract}
Anaesthetic management of an extremely low birth weight (ELBW) neonate is always a challenge. Challenges are substantial beginning from pre-operative preparation till the neonate has been shifted to the neonatal intensive care unit. We report a case of an ELBW neonate who was operated twice for necrotising enterocolitis on day thirteen and day nineteen of life, weighing 815 grams and 746 grams respectively. Perioperative management and repair of the perforation due to necrotising enterocolitis was done on two occasions owing to the recurrence in a neonate with multiple co-morbidities. A planned approach and appropriate preparation led to a smooth peri-operative course both the times. This report discusses the multiple anaesthetic challenges and emphasises the role of a closed loop functioning among paediatric anaesthesiologists, neonatologists and the paediatric surgeons.
\end{abstract}

Keywords: Co-morbidities, Extremely low birth weight, Infant, Neonate, Necrotising enterocolitis, Perioperative management.

\section{Introduction}

WHO statistics states that across 184 nations, incidence of babies born prematurely varies between $5 \%-18 \% .^{1}$ ELBW babies or micropremies are the ones with the birth weight of less than 1000 grams. Though with improved medical care, the mortality rate in this patient population has drastically reduced, especially at a tertiary care centre (survival rate $75-80 \%$ for $600-$ 700 grams, $85 \%$ for $700-800$ grams and $90 \%$ for 800 - 1000 gram babies), the morbidity continues to be the same. ${ }^{2}$ Whereas the primary causes of death are pulmonary haemorrhage $(25 \%)$, respiratory distress syndrome (22.5\%), intraventricular haemorrhage $(22.5 \%)$ and sepsis $(20 \%)$, commonest complications that often require surgery are patent ductus arteriosus (PDA) and necrotising enterocolitis (NEC). ${ }^{3}$ We report a case of ELBW neonate who had presented with these multiple co - morbidities, for peri-operative management for an enteric perforation repair.

\section{Case report}

A female child born at 29 weeks of gestation with birth weight 840 grams was diagnosed to have hyaline membrane disease, for which surfactant was administered. Nasal Continuous Positive Airway Pressure (CPAP) was instituted but over next few days baby developed pneumonia, haemolytic jaundice, hypoglycaemia (requiring glucose infusion rate of 10 $\mathrm{mg} / \mathrm{kg} / \mathrm{min}$ ), dyselectrolytemias, septicaemia and septic shock, and intraventricular haemorrhage (IVH). She was started on inotropes (Inj Dopamine and Dobutamine) on day five of life, along with appropriate antibiotics and Vitamin K. By ninth day of life, abdominal distension was noticed, which deteriorated over next 24 hours and NEC was diagnosed. Septic shock resolved but after initial conservative management for NEC, it was decided to take up the patient for surgery. Paediatric anaesthesia team visited the neonatal intensive care unit (NICU) along with the surgeon a day prior to the surgery on day 13 of life (weight 815 grams). All details of management were discussed thoroughly with the neonatologist, resident as well as the staff nurse in charge of the case. Peripheral venous access was secured with a peripherally inserted central catheter (PICC) at right cubital fossa and a $24 \mathrm{G}$ cannula in the left foot, in the NICU. An intra arterial catheter $(24 \mathrm{G})$ was secured in posterior tibial artery. Complete blood count, coagulation profile, serum electrolytes, arterial blood gas (ABG) and blood sugar were advised to be done two hours prior to surgery. A six member team shifted the patient from NICU to operation theatre (ambient temperature 27 degree Celsius) in a paediatric incubator. Intra-operative monitoring was instituted that included electrocardiography (ECG), pulse oximetry $\left(\mathrm{SpO}_{2}\right)$, temperature, end tidal carbon dioxide $\left(\mathrm{EtCO}_{2}\right)$, invasive blood pressure and urine output. Adequate measures were taken for temperature homeostasis like wrapping extremities, head and chest with plastic sheets, use of warming pad, administering warm intravenous (IV) fluids, and use of warm and humidified inspired gases.

Induction was done with $20 \mu \mathrm{g} / \mathrm{kg}$ fentanyl IV followed by intubation with $50 \mu \mathrm{g} / \mathrm{kg}$ Atracurium. Trachea was intubated with size 2.0 Endo-Tracheal Tube (ETT) which was fixed at $7 \mathrm{~cm}$ mark at lip, with adhesive tape over a layer of Tegaderm $^{\mathrm{TM}}$ on the cheeks. Anaesthesia was maintained with 1.5-2\% Sevoflurane with mixture of oxygen and air. Intra- 
operative IV fluids were administered using two infusion pumps. Maintenance fluid administered was Ringer's lactate with $2.5 \%$ Dextrose at rate of $7.5 \mathrm{ml} / \mathrm{hr}$ $(180 \mathrm{ml} / \mathrm{kg} /$ day $)$ and replacement fluid used was Ringer's lactate at the rate of $16 \mathrm{ml} / \mathrm{hr}$. Intra-operative $\mathrm{SpO}_{2}$ was maintained between $87 \%$ and $94 \%$. Heart rate varied between 130 and 150 beats/min. Lowest mean arterial pressure recorded was $32 \mathrm{mmHg}$ which responded to Packed Red Blood Cell infusion $(20 \mathrm{ml})$. Intra-operative urine output was adequate. Random blood sugar was $104 \mathrm{mg} / \mathrm{dl}$ (20 minutes after the start of surgery) and $118 \mathrm{mg} / \mathrm{dl}$ (on culmination of surgery). Intra-operative and immediate post-operative ABGs were within normal limits. Core body temp was maintained between 35 and 36 degree Celsius using a nasopharyngeal probe.

Post surgery, patient was shifted to NICU for postoperative ventilation and was extubated in $48 \mathrm{~h}$. There was a recurrence of perforation within one week for which patient was re-operated successfully on day nineteenth day of life (weight 746 grams). The same anaesthetic protocol was followed and patient was shifted to NICU for post-operative care, second time as well.

\section{Discussion}

ELBW babies may present postnataly for surgery due to the multiple congenital anomalies including PDA, retinopathy of Prematurity (ROP), NEC, and IVH with hydrocephalous and hernias. What makes the perioperative management extremely challenging is the plethora of anaesthetic implications with respect to multiple co-morbidities, clubbed with the anatomical and physiological immaturity of various systems. Flip flop circulation is very common in presence of hypoxia, hypercarbia, acidosis and hypothermia which may also lead to pulmonary hypertension, whereas hyperoxia may cause bronchopulmonary dysplasia, ROP and neurodevelopmental abnormalities. Therefore, in our case we aimed at the target oxygen saturation between $87-94 \%$. Incidence of hypoglycaemia is very high in ELBW babies therefore; intra-operative euglycemia was maintained with background infusion of the glucose. Evidence shows that hyperglycemia not only leads to osmotic diuresis with consequent electrolyte disturbances but may also be associated with hypoxic ischemic brain and spinal cord injury. ${ }^{4}$ Thermoregulation is a critical issue in these neonates as heat loss is 15 times more than that in full term babies. All possible steps, were taken to avoid hypothermia like wrapping the baby in the plastic sheets, maintaining the OT ambient temperature, use of warm blankets and in line warming system for fluids. Hypothermia has been associated with IVH and death in this subset of neonates. ${ }^{5}$

Central nervous system injury caused by periventricular leukomalacia (leading to cerebral palsy subsequently) and IVH is a major concern in these
ELBW neonates. ${ }^{6}$ Common insults like hypoxemia, hypotension and inflammation in this age group effects adversely the immature oligodendrocyte precursors leading to periventricular leukomalacia. Inadequate analgesia has been known to cause and deteriorate IVH, which justifies the liberal use of analgesia without compromising haemodynamics. Antibiotics directly kill gut flora, thereby, increasing susceptibility to gastrointestinal bleed. Vitamin $\mathrm{K} \quad(0.2 \mathrm{mg} \quad \mathrm{IM})$ was administered in NICU prior to the surgery, to reduce intra-operative risk of bleeding. Serum electrolytes and haemoglobin (minimum $10 \mathrm{gm} / \mathrm{dl}$ ) should be within acceptable limits. Peri-operative IV fluid management is an issue of great concern. Two separate IV accesses should be available, one for maintenance fluids (crystalloid with 1-2.5\% Dextrose) and the other for replacement fluids (crystalloids, fresh frozen plasma, blood, platelets). Maintenance fluid, if not wisely balanced is bound to cause either intravascular hypotonia leading to hyponatremia at one end or hypoglycemia on the other. Crystalloids at the rate of $10-50 \mathrm{ml} / \mathrm{kg} / \mathrm{hr}$ may be required to compensate for insensible losses from the exposed bowel in NEC.

Induction can be done using Sevoflurane (MAC < 2), Halothane (MAC < 0.55) or Fentanyl $(10-50$ $\mu \mathrm{g} / \mathrm{kg}$ ). ${ }^{7}$ Opioids in adequate doses provide more stable haemodynamics. Lower induction doses of Fentanyl $(10-20 \mu \mathrm{g} / \mathrm{kg})$ should be administered to infants with increased intra-abdominal pressure, since the elimination half-life is predictably prolonged. $^{8}$ Atracurium or Cis atracurium is a preferred muscle relaxant in wake of immature liver functions. Adhesive tapes used for fixing ETT may strip off epidermis when removed. The traumatic effect of adhesive removal has been documented on premature infants and includes reduced barrier function, increased trans-epidermal water loss, increased permeability, erythema and skin stripping. ${ }^{9}$ Therefore, pectin based or hydrocolloid adhesives should be used between skin and other adhesives.

Apart from minimum mandatory monitoring invasive blood pressure should be monitored since, the blood pressure of preterm infants is normally closer to the lower auto-regulatory limit of cerebral circulation, especially in ELBW babies. ${ }^{10}$ Beat to beat assessment of blood pressure (BP) would assist in immediate optimisation of the same if required. $\mathrm{ABG}$, serum electrolytes and blood sugar can be measured easily intra-operatively with the invasive BP monitoring.

Transportation of these patients post-operatively to NICU requires meticulous planning and preparation. The problem gets compounded with all the IV cannula, infusions and ETT in situ, this poses a significant challenge especially when the baby is sick and unstable. In fact, if possible surgeries in this age group are safe to be performed in the NICU. ${ }^{11}$ Also, when post-operative extubation is planned in such a surgery, preoperative use of caffeine $(10 \mathrm{mg} / \mathrm{kg})$, non- Fentanyl-induction 
approach and use of caudal analgesia is recommended. Since in our index case the child was in sepsis with enteric perforation the decision of elective ventilation was planned. To conclude finally, what seems to be indispensible in this case management is thorough knowledge of the subject and the team work, which includes flawless coordination, meticulous planning and preparation among paediatric anaesthesiologist, neonatologist and the paediatric surgeon.

\section{Support/Source: Nil}

\section{Conflict of Interest: Nil}

\section{References}

1. Blencowe H, Cousens S, Oestergaard M, Chou D, Moller AB, Narwal R, Adler A, Garcia CV, Rohde S, Say L, Lawn JE. National, regional and worldwide estimates of preterm birth. The Lancet, June 2012. 9; 379(9832):2162-72.

2. Ogawa M, MatsudaY, Kanda E, Konno J, Mitani M, Makino Y, and Matsui H. Survival rate of extremely low birth weight infants and its risk factors: Case control study in Japan. ISRN Obtetrics and Gynaecology 2013; ID 873563: 6 pages. http://dx.doi.org/10.1155/2013/873563.

3. Tagore A et al. Mortality and morbidity in extremely low birth weight (ELBW) infants in a neonatal intensive care unit. Indian J Pediatrics 2013;80(1):1620.

4. Murat I, Dubois MC. Perioperative fluid therapy in Pediatrics. Pediatric anesthesia 2008;18:363-70.

5. Miller SS, Lee HC, Gould JB. Hypothermia in very low birth weight infants: distribution, risk factors and outcomes. Journal of Perinatology 2011;31:549-56.

6. Eichenwald EC, Stark AR. Management and outcomes of very low birth weight baby. N Engl J Med 2008;358:1700-11.

7. Gregory GA and Andropoulos DB. Gregory's Pediatric Anesthesia 2012; $5^{\text {th }}$ edition: 475-96.

8. Kinouchi K. Anaesthetic considerations for the management of very low and extremely low birth weight infants. B Practice \& Research Clinical Anaesthesiology 2004;18(2):273-90.

9. Lund C, Nonato L, Kuller J, et al. Disruption of barrier function in neonatal skin associated with adhesive removal. J Pediatr 1997;131:367-72.

10. Davis PJ, Cladis FP, Motoyama EK. Smith's Anesthesia for Infants and Children 2010; $8^{\text {th }}$ edition:531.

11. Sinha SK, Negi S. Bedside neonatal intensive care unit surgery - Myth or Reality! Journal of neonatal surgery 2013;2(2):20. 